\title{
Síndrome de burnout na equipe de enfermagem em unidade de terapia intensiva neonatal: revisão integrativa
}

\author{
Burnout syndrome in the nursing team in a neonatal intensive care unit: an integrative review \\ Síndrome de burnout en el equipo de enfermería en unidad de terapia intensiva neonatal: una revisión integrativa \\ Natália Conteçote Russo ${ }^{1}$ (D) https://orcid.org/0000-0002-7763-0524
}

\section{Resumo}

Objetivo: Identificar a produção científica referente à síndrome de burnout na equipe de enfermagem em unidades de terapia intensiva neonatal e buscar evidências de alternativas que amenizem e/ou solucionem seus efeitos. Métodos: Trata-se de uma revisão integrativa da literatura realizada nas bases de dados Scielo, Lilacs, Medline, BDenf e PubMed, no período compreendido entre 2008 e 2017.

Resultados: Foram selecionados quatro artigos. Observa-se que as publicações sobre o tema nos últimos dez anos são escassas e evidenciam a prevalência da síndrome de burnout na equipe de enfermagem em unidades de terapia intensiva neonatal.

Conclusão: Destacou-se a necessidade da realização de pesquisas que colaborem com evidências científicas que abordem alternativas para amenizar e/ou solucionar os sinais do esgotamento profissional.

\section{Abstract}

Objective: To identify the scientific literature on the role of the burnout syndrome in the nursing team in neonatal intensive care units and to seek evidence of alternatives that minimize and/or solve their effects.

Methods: This is an integrative literature review conducted in the databases Scielo, Lilacs, Medline, BDenf e PubMed, in the period between 2008 and 2017.

Results: Four articles were included in the study. It is observed that the studies on the subject in the last ten years are scarce and demonstrate the prevalence of the burnout syndrome in the nursing team in neonatal intensive care units.

Conclusion: It is necessary to carry out research that provide scientific evidence that address alternatives to improve and/or solve the signs of professional burnout.

\section{Resumen}

Objetivo: Identificar la literatura científica sobre el papel del síndrome de burnout en el equipo de enfermería en unidades de terapia intensiva neonatal y buscar evidencias de alternativas que amenizen $\mathrm{y} / 0$ solucionen sus efectos.

Métodos: Se trata de una Revisión de la de la literatura integrativa llevó a cabo en las siguientes bases de datos Scielo, Lilacs, Medline, BDenf e PubMed, en el periodo comprendido entre 2008 e 2017.

Resultados: Se seleccionaron cuatro artículos, señalando que las publicaciones sobre el tema en los últimos diez años son escasas, afirmando la prevalencia del síndrome de burnout en el equipo de enfermaría en unidades de cuidados intensivos neonatales.

Conclusión: Se destacó la relevancia de la necesidad de llevar a cabo una investigación que colabore con evidencia científica que aborden alternativas para amenizar y/o solucionar los signos del agotamiento profesional.

\section{Descritores}

Esgotamento profissional; Unidades de terapia intensiva neonatal; Enfermagem; Enfermagem pediátrica

Keywords

Burnout professional; Neonatal Intensive care units; Nursing; Pediatric nursing

\section{Descriptores}

Agotamiento profesional; Unidades de cuidado intensivo neonatal; Enfermería; Enfermería pediátrica

\section{Como citar:}

Russo NC. [Burnout syndrome in the nursing team in a neonatal intensive care unit: an integrative review]. Rev Soc Bras Enferm Ped. 2020;20(1):40-6. Portuguese

\footnotetext{
${ }^{1}$ Faculdade de Medicina de Botucatu, Universidade Paulista Júlio de Mesquita Filho, Botucatu, SP, Brasil.

Conflitos de interesse: nada a declarar.

Submetido: 6 de Dezembro de 2017 | Aceito : 29 de Junho de 2020

Autor correspondente: Natália Conteçote Russo | E-mail: nataliarusso@outlook.com

DOI: http://dx.doi.org/10.31508/1676-3793202000006
} 


\section{Introdução}

Em um ambiente hospitalar, a Unidade de Terapia Intensiva Neonatal (UTIN) pode ser uma fonte geradora de estresse na equipe de enfermagem, devido às características do setor, que é uma unidade fechada, com muitos ruídos e com tecnologias específicas, e devido ao cuidado de recém-nascidos criticamente enfermos, que são altamente vulneráveis e necessitam de cuidados de enfermagem especiais e contínuos. ${ }^{(1)}$

As enfermeiras e demais profissionais da área da saúde que atuam em unidade neonatal precisam de boas condições de integridade física e mental para poder oferecer atendimento pleno às demandas apresentadas pelos recém-nascidos. Para isso, precisam de uma adequada integração entre corpo e mente. ${ }^{(2)}$

Uma das doenças relacionadas diretamente ao processo de trabalho recebe a denominação de síndrome de burnout. Esta síndrome tem sido descrita como resultante da vivência profissional em um contexto de relações sociais complexas, envolvendo a representação que a pessoa tem de si e dos outros. Um trabalhador que antes se mostrava muito envolvido afetivamente com os seus pacientes ou com o trabalho em si, desgasta-se, e, em um dado momento, desiste, perde a energia ou se "queima" completamente. ${ }^{(3)}$

Burn significa queimar e out significa exterior, esgotamento. Burnout indica que o esgotamento do profissional já extrapolou os limites admissíveis. De forma geral, representa algo que se queimou completamente, deixando de funcionar por absoluta falta de energia. O burnout é uma resposta de defesa, mesmo que imprópria, à cronificação do estresse ocupacional que chegou a limites intoleráveis. ${ }^{(4)}$

Atualmente, já é consensual que o burnout é uma resposta ao estresse crônico vivenciado no ambiente de trabalho. No entanto, deve-se atentar para não confundir esses dois construtos e nem os tratar como sinônimos. ${ }^{(5)}$

O burnout é entendido como um conceito multidimensional que envolve três componentes: 1) exaustão emocional, que compreende sentimentos de fadiga e esgotamento energético emocional devidos à percepção do indivíduo de ser exigido emocionalmente mais do que ele pode tolerar; 2) despersonalização, que está associada às atitudes negativas de distanciamento excessivo e dureza na relação dos profissionais com os beneficiários do seu serviço; e 3) diminuição da realização pessoal (esse fator refere-se a sentimentos de incompetência nas atividades realizadas). Essas dimensões retratam, respectivamente, os aspectos individual, interpessoal e de autoavaliação do burnout.(5)

As altas taxas de burnout entre os enfermeiros em geral estão associadas a muitos efeitos adversos no atendimento ao paciente, incluindo aumento das infecções associadas aos cuidados de saúde, diminuição no reconhecimento e notificação de erros, aumento na mortalidade de pacientes e diminuição da satisfação profissional. $^{(6,7)}$

Os hospitais cujos enfermeiros têm altas taxas de burnout são mais propensos a ter altas taxas de atraso e absenteísmo dos funcionários. ${ }^{(8)}$

Ressalta-se a importância de uma assistência humanizada dentro dessa unidade, que é um ambiente complexo e um gerador de estresse não só para os bebês, mas também para os pais e para os profissionais. Sobre isso, compreendemos que a humanização não envolve apenas os bebês internados, mas também os pais e os profissionais que atuam nesse ambiente. Por isso, a humanização pode ser um desafio muito difícil para uma pessoa com esgotamento profissional. ${ }^{(9)}$

O conhecimento sobre esta síndrome e sobre as estratégias para preveni-la e alternativas para amenizar e/ou solucionar seus efeitos é essencial para a gestão dos serviços de saúde, para que se ofereça uma assistência humanizada, com profissionais que trabalhem motivados, e um cuidado efetivo e humanizado, minimizando os riscos relacionados à segurança do paciente.

Assim, questionou-se nesta pesquisa: como a literatura científica tem abordado a síndrome de burnout na equipe de enfermagem em unidades de terapia intensiva neonatal?

Dessa forma, buscou-se conhecer a produção do conhecimento acerca da síndrome de burnout na equipe de enfermagem em unidades de terapia intensiva neonatal e as evidências de alternativas que amenizem e/ou solucionem seus efeitos.

\section{Métodos}

Para alcançar o objetivo proposto nesse estudo, o método escolhido foi a revisão integrativa da literatura, 
que tem a finalidade de reunir e sintetizar os resultados de pesquisas sobre um delimitado tema, de maneira sistemática e ordenada, contribuindo para o aprofundamento do conhecimento a respeito do tema investigado e permitindo a síntese de múltiplos estudos publicados e a elaboração de conclusões gerais a respeito de uma particular área de estudo. ${ }^{(10)}$

Esse trabalho apresentará a descrição das seis fases percorridas para a elaboração da revisão de literatura integrativa, baseadas em Souza, Silva e Carvalho. ${ }^{(11)}$

\section{$1^{\text {a }}$ Fase: Elaboração da pergunta norteadora}

A partir da questão "como a literatura científica tem abordado a síndrome de burnout na equipe de enfermagem em unidades de terapia intensiva neonatal?", serão utilizadas as expressões "Burnout", "Esgotamento Profissional" e "Unidade de Terapia Intensiva Neonatal", todas ligadas ao operador booleano "AND". A escolha desses descritores foi baseada nas expressões do DeCS (Descritores em Ciências da Saúde), um tipo de dicionário criado pelo Centro Latino-Americano e do Caribe de Informação em Ciências da Saúde, mais conhecido pela sigla BIREME (de sua denominação original: Biblioteca Regional de Medicina), para servir como uma linguagem única (padrão) na indexação de publicações, facilitando a recuperação de assuntos comuns da literatura científica.

A busca foi realizada em cinco bancos de dados para a comunicação científica utilizados em pesquisas: SCIELO (Scientific Eletronic Library Online), LILACS (Literatura Latino-Americana e do Caribe em Ciências da Saúde), MEDLINE (Medical Literature Analysis and Retrieval System Online), BDENF (Base de dados da Enfermagem) e PUBMED (U.S. National Library of Medicine).

\section{$2^{\mathrm{a}}$ Fase: Busca ou amostragem na literatura}

A busca na literatura deve ter uma seleção criteriosa das pesquisas. Este levantamento bibliográfico preliminar pode ser entendido como um estudo exploratório, com a finalidade de proporcionar familiaridade do pesquisador com a área de estudo na qual está interessado. Para esse fim, serão acessadas as bases de dados citadas acima, que possuem acesso eletrônico. ${ }^{(11)}$

\section{$3^{\mathrm{a}}$ Fase: Coleta de dados}

Para a seleção dos artigos, os critérios de inclusão definidos foram: artigos na íntegra, publicados em língua portuguesa, inglesa ou espanhola, publicados e/ou indexados nas bases de dados nos últimos dez anos, referidos no período de 2008 a 2017, abordando assuntos relacionados à síndrome de burnout na equipe de enfermagem em unidade de terapia intensiva neonatal e com acesso gratuito.

\section{$4^{\mathrm{a}}$ Fase: Análise crítica dos estudos incluídos}

Nessa fase, aborda-se de forma organizada o rigor e as características de cada artigo, buscando observar as suas convergências e divergências na comparação com os demais achados.

\section{$5^{\mathrm{a}}$ Fase: Discussão dos resultados}

Nessa fase é realizada a interpretação e síntese dos resultados, comparando os dados evidenciados na análise de cada artigo e identificando as possíveis lacunas no conhecimento sobre o tema, de forma a delimitar prioridades para futuras pesquisas.

\section{$6^{\text {a }}$ Fase: Apresentação da revisão integrativa}

Para facilitar a análise dos artigos selecionados, os estudos foram apresentados em um quadro e os artigos foram categorizados em função das variáveis analíticas, compreendendo os seguintes aspectos: autoria e ano de publicação, local do estudo, tipo de estudo, número de amostras, instrumentos utilizados na pesquisa e principais resultados.

Com a utilização do instrumento de tabulação elaborado, foi dado seguimento nas técnicas de extração de dados das fontes primárias, possibilitando resumir e organizar os achados.

\section{Resultados e Discussão}

A partir da primeira busca nas bases de dados estabelecidas, obtiveram-se 74 estudos. Foi realizada a lei- 
tura de todos os títulos e resumos, respeitando-se os critérios de inclusão e exclusão. Assim, apenas quatro estudos foram selecionados para análise detalhada. A seguir, a Figura 1 ilustra uma síntese do processo de seleção de artigos.

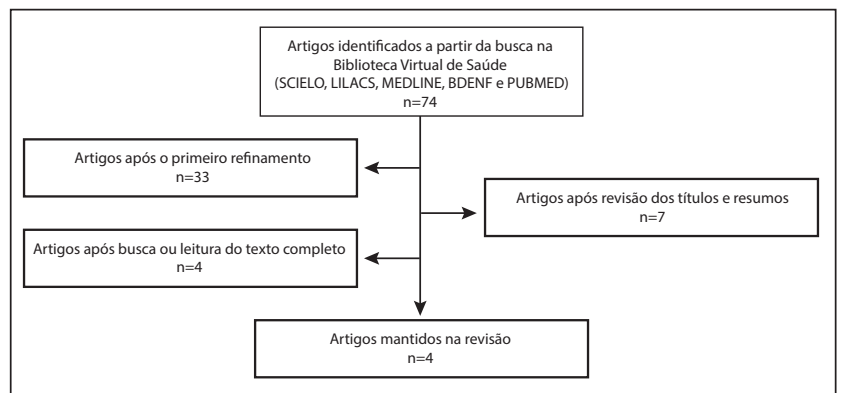

Figura 1. Fluxograma de identificação das etapas de seleção dos artigos para a revisão integrativa

Conforme apresentado na figura, foi observada uma pequena quantidade de estudos acerca do tema na literatura científica. A base de dados SCIELO não retornou nenhum resultado; quatro artigos foram obtidos na LILACS, 29 na MEDLINE, apenas um na BDENF e 40 na PUBMED.

Após o primeiro refinamento seguindo os critérios de inclusão, obteve-se o total de 33 artigos. Ao realizar uma revisão dos títulos e resumos dos trabalhos, foram excluídos vinte e seis estudos que não abordavam o tema em questão, ficando apenas sete estudos.

Após a leitura dos textos completos, foram excluídos mais três trabalhos que não atendiam aos critérios de inclusão por não abordarem a síndrome de burnout na equipe de enfermagem em uma unidade de terapia intensiva neonatal. Dessa forma, foram selecionados apenas quatro estudos, três realizados nos Estados Unidos e um no Irã.

O quadro abaixo apresenta os artigos selecionados para esse estudo, assim como os autores, ano de publicação, país de origem, tipo de estudo, número de amostras, instrumentos utilizados na pesquisa e os principais resultados obtidos (Quadro 1).

Três artigos publicados nos Estados Unidos foram encontrados na busca dessa revisão integrativa. Esses artigos abordaram o mesmo estudo, realizado em 44 unidades neonatais que aceitaram participar. Dois foram publicados em 2014 e um em 2017, e todos constataram a presença de burnout na equipe multidisciplinar em diferentes perspectivas. ${ }^{(12-14)}$

O burnout é gerador de importantes perdas de recursos humanos e econômicos, podendo levar os profissionais ao afastamento laboral de curto e médio prazo, por ansiedade, depressão, transtornos do estresse, transtornos psicossomáticos e até mesmo ideação suicida. ${ }^{(16)}$

Isso ocorre quando as tensões causadas pelo trabalho não são adequadamente reconhecidas e enfrentadas. Assim, o indivíduo passa a apresentar autoconceito negativo, atitudes negativas em relação ao trabalho e perda de interesse pelos usuários de seus serviços. ${ }^{(17)}$

A solução encontrada pelas organizações é o desligamento de colaboradores, e não o enfrentamento da síndrome em si. É necessário que a gerência de enfermagem conheça a situação que seus colaboradores estão vivenciando, para que as organizações possam adotar estratégias para ajudar o trabalhador a lidar com o estresse, tais como campanhas informativas,

Quadro 1. Distribuição dos estudos segundo base de dados, título dos artigos, autores, ano de publicação, método adotado, país de origem e resultados da pesquisa

\begin{tabular}{|c|c|c|c|c|c|c|}
\hline Autores & $\begin{array}{c}\text { Ano de } \\
\text { publicação }\end{array}$ & País & Tipo de estudo & N. ${ }^{\circ}$ de Amostras & Instrumentos & Resultados \\
\hline Sexton, et al. ${ }^{(12)}$ & 2014 & $\begin{array}{l}\text { Estados } \\
\text { Unidos }\end{array}$ & Transversal & $\begin{array}{l}2073 \text { funcionários } \\
\text { de Unidade } \\
\text { Neonatal }\end{array}$ & $\begin{array}{c}\text { Inventário Maslach } \\
\text { Burnout }\end{array}$ & $\begin{array}{l}\text { A relação entre o feedback da técnica de WalkRounds e a } \\
\text { diminuição do burnout é importante devido à escassez de } \\
\text { intervenções eficazes para a prevenção desta síndrome. }\end{array}$ \\
\hline Profit, et al. ${ }^{(13)}$ & 2014 & $\begin{array}{l}\text { Estados } \\
\text { Unidos }\end{array}$ & Transversal & $\begin{array}{l}2073 \text { funcionários } \\
\text { de Unidade } \\
\text { Neonatal }\end{array}$ & $\begin{array}{c}\text { Inventário Maslach } \\
\text { Burnout }\end{array}$ & $\begin{array}{l}\text { Mostra uma alta prevalência de burnout entre o pessoal } \\
\text { da UTI Neonatal, especialmente entre enfermeiros, } \\
\text { profissionais de enfermagem e prestadores de cuidados } \\
\text { respiratórios. }\end{array}$ \\
\hline Soroush, et al. ${ }^{(14)}$ & 2016 & Irã & Transversal & $\begin{array}{l}86 \text { enfermeiros } \\
\text { de UTI Neonatal }\end{array}$ & $\begin{array}{c}\text { Inventário Maslach } \\
\text { Burnout }\end{array}$ & $\begin{array}{l}0 \text { escore médio de burnout foi moderado no domínio } \\
\text { da exaustão emocional, forte no domínio da falta de } \\
\text { desempenho e fraco em despersonalização. }\end{array}$ \\
\hline Tawfik, et al. ${ }^{(15)}$ & 2017 & $\begin{array}{l}\text { Estados } \\
\text { Unidos }\end{array}$ & Transversal & $\begin{array}{l}2073 \text { funcionários } \\
\text { de Unidade } \\
\text { Neonatal }\end{array}$ & $\begin{array}{c}\text { Inventário Maslach } \\
\text { Burnout }\end{array}$ & $\begin{array}{l}\text { Prevalência de burnout é maior entre os não médicos, } \\
\text { trabalhadores do turno do dia e trabalhadores mais } \\
\text { experientes. }\end{array}$ \\
\hline
\end{tabular}


incentivo aos valores humanos voltados ao trabalho, projetos de ergonomia e relaxamento, boas condições de trabalho, e combate a sobrecargas de atividades. ${ }^{(18)}$

Um dos artigos encontrado nessa revisão, realizado nos Estados Unidos, avaliou se as atividades de Leadership WalkRounds seriam uma ferramenta para melhorar a segurança do paciente em cuidados neonatais e melhorar o trabalho em equipe, diminuindo o desgaste no trabalho e a síndrome de burnout. Esse estudo constatou que há uma escassez de intervenções eficazes para reduzir o burnout nos trabalhadores, mas que o feedback destas atividades diminui esta síndrome, visto que proporciona um melhor ambiente e um melhor clima de trabalho em equipe, reduzindo o desgaste e o estresse na unidade de terapia intensiva neonatal. ${ }^{(12)}$

Leadership WalkRounds é um método amplamente utilizado em hospitais norte-americanos e consiste num programa de visitas às linhas de frente da organização para observar e conversar com os funcionários enquanto executam seu trabalho. A intenção é fazer com que a alta direção e os funcionários que atuam na linha de frente trabalhem juntos para identificar e resolver obstáculos à eficiência, à qualidade ou à segurança. ${ }^{(19)}$

Um estudo que comparou instituições para avaliar se ambientes desfavoráveis à prática profissional da equipe de enfermagem podem resultar no desenvolvimento da síndrome de burnout destacou que os resultados da sua pesquisa podem ser utilizados por gerentes de enfermagem na implementação de mudanças no ambiente hospitalar, com o objetivo de proporcionar aos enfermeiros o exercício de uma prática com autonomia, controle sobre o ambiente e boas relações com os médicos, contribuindo para alcançar resultados positivos para os pacientes, profissionais e instituições. ${ }^{(20)}$

Os principais fatores que influenciam diretamente no desenvolvimento do esgotamento profissional estão relacionados a questões gerenciais, como: escassez de pessoal, que leva ao acúmulo de tarefas; trabalho por turno; excesso de horas extras; conflitos com demais colegas que compõem a equipe; inexistência de plano de cargos e salários; e má remuneração. ${ }^{(21)}$

A gerência de enfermagem das instituições deve conhecer as medidas preventivas e o tratamento desta síndrome, que precisa ser abordada como proble- ma coletivo e organizacional, e não como um problema individual. ${ }^{(22)}$

Uma pesquisa que identificou os fatores de risco para a síndrome de burnout refere ainda que debates sobre o assunto devem ser realizados não só entre líderes de trabalhadores, mas também com profissionais de recursos humanos, sindicatos, organizações, advogados, juízes, psicólogos, médicos e ministério público, a fim de buscar alternativas que possam sanar ou diminuir os riscos de desenvolvimento do problema. ${ }^{(23)}$

Também é necessário ressaltar a necessidade de políticas públicas específicas para o enfrentamento e prevenção do estresse e burnout. Muitos profissionais não têm conhecimento acerca dos direitos trabalhistas relacionados ao adoecimento organizacional, o que corrobora com os estudos acima no que tange à necessidade de reflexão acerca do estresse ocupacional nas organizações. ${ }^{(18)}$

Outro estudo teve como objetivos testar as propriedades psicométricas de uma pequena escala de burnout de quatro itens da escala de exaustão emocional do Inventário de Burnout de Maslach e realizar uma avaliação comparativa entre o burnout e a capacidade de resiliência dos cuidadores em unidades de terapia intensiva neonatal. Esse estudo mostrou uma alta prevalência de burnout entre os profissionais da unidades de terapia intensiva neonatal, especialmente enfermeiros, profissionais da enfermagem e prestadores de cuidados respiratórios. ${ }^{(13)}$

Considerando a exaustão emocional e o desânimo da pessoa acometida por burnout, é extremamente importante trabalhar a resiliência do indivíduo frente a conteúdos emocionais aflorados que possam dificultar a dinâmica de resolução de problemas. A resiliência envolve não somente a capacidade de lidar com as mais diversas situações, mas também a habilidade de se adaptar às mudanças que podem ocorrer dentro do ambiente em que se está inserido, para que se lide com a pressão a partir de uma visão mais clara e racional, sem desconsiderar os componentes emocionais envolvidos, mas trabalhando para não ser dominado pelos mesmos. Dessa forma, trabalha-se a racionalidade e a emocionalidade, focando no problema e não nas emoções. ${ }^{(24)}$

Uma pesquisa realizada em um hospital do sul do Brasil apontou que a equipe de enfermagem relata dificuldades relacionadas à falta de planejamento de 
ações que viabilizem a prática do cuidado humanizado dentro do ambiente de trabalho, como o apoio ao profissional e o estímulo à motivação. São necessárias ações com os profissionais, tais como momentos de reflexão com a equipe e atuação do serviço de psicologia, de forma a apoiar os profissionais do setor e estimular a motivação. ${ }^{(25)}$

Outro estudo, publicado em 2017, buscou analisar a prevalência do burnout em unidades de cuidados intensivos neonatais na Califórnia e testar a relação entre o burnout e a prevalência de infecção associada aos cuidados de saúde em recém-nascidos de muito baixo peso. A principal descoberta desse estudo foi que a prevalência da síndrome de burnout é maior entre os profissionais não médicos, trabalhadores do turno do dia e com cinco anos ou mais de experiência na área. Além disso, a percepção de trabalhar muito é associada ao aumento de infecção associada aos cuidados de saúde. ${ }^{(15)}$

Para que atuem de forma segura, sem que haja riscos de ocorrência de eventos adversos ao paciente, os trabalhadores de enfermagem precisam manter $\mathrm{o}$ equilíbrio físico e psicológico. Entretanto, a ocorrência de estresses físicos e emocionais relacionados a processos de trabalho que esses profissionais podem vivenciar, como situações de sofrimentos, pode dificultar ou até mesmo impossibilitar uma atuação com segurança nos cuidados prestados. ${ }^{(26)}$

Estudos evidenciam a relação entre burnout e segurança do paciente, haja vista que um nível de burnout mais alto esteve associado a um baixo grau de segurança do paciente e, em contrapartida, uma cultura de segurança do paciente positiva esteve correlacionada com um baixo nível de burnout. . $^{(27,28)}$

O outro trabalho encontrado foi realizado no Irã e teve como objetivo investigar a relação entre o desgaste e a competência clínica das enfermeiras nas unidades de terapia intensiva neonatais. Nesse estudo, o Inventário de Burnout de Maslach foi aplicado em 86 enfermeiros, e os resultados mostraram uma associação inversa entre burnout do trabalho e competência clínica, de modo que quanto maior a competência clínica do enfermeiro, menor a probabilidade de desenvolvimento da síndrome de burnout nas equipes e vice-versa. $^{(14)}$

A falta de autoconfiança e uma base de conhecimento inadequada são fatores que contribuem com a tensão adicional no processo de tomada de decisão, o que sugere que indivíduos que não possuem a experiência de vida profissional necessária tornam-se mais predispostos à síndrome de burnout. ${ }^{(29)}$

Em contrapartida, estudo comprova que as pessoas de maior nível educacional possuem maior propensão ao burnout. Isso pode ser explicado pelo fato de o profissional possuir baixa realização pessoal no trabalho e não acreditar que pode obter ascensão profissional, devido a características inerentes à profissão e à instituição. ${ }^{(30)}$

Com essa revisão integrativa da literatura em relação à síndrome de burnout na equipe de enfermagem em unidades de terapia intensiva neonatal, podemos perceber uma escassez de trabalhos publicados internacionalmente. Nos últimos dez anos, não há nenhum trabalho publicado no Brasil com profissionais da enfermagem dessas unidades acerca do assunto e, no geral, são poucos os trabalhos publicados.

Devido a pequena amostra de artigos encontrados nessa revisão integrativa, houve uma limitação dos resultados esperados de acordo com os objetivos propostos, falta de dados relacionados à identificação de dificuldades na detecção ou prevenção do esgotamento profissional na equipe de enfermagem em unidade de terapia intensiva neonatal e falta de evidências científicas que abordem as alternativas para solucionar sinais da síndrome de burnout.

\section{Considerações finais}

O esgotamento profissional e a síndrome de burnout destacam-se dentre as doenças ocupacionais mais comuns na equipe de enfermagem que trabalha em unidade de terapia intensiva neonatal, devido a características inerentes à profissão e à especificidade de seu trabalho. A presente pesquisa teve como objetivo conhecer o panorama de publicações sobre o esgotamento profissional entre esses trabalhadores, oferecendo contribuições importantes para a caracterização da produção científica internacional acerca dos conceitos em questão. Fizeram parte da revisão integrativa apenas quatro estudos, representando menos de $6 \%$ do total inicial encontrado $(n=74)$. Verificou-se que as publicações sobre o tema nos últimos dez anos são escassas e que todas evidenciam a presença da síndrome 
de burnout na equipe de enfermagem em unidades de terapia intensiva neonatal. Observou-se que ainda são incipientes na literatura as evidências de estratégias de enfrentamento/intervenções adotadas por instituições, gestores ou líderes para prevenir a síndrome de burnout em trabalhadores da equipe de enfermagem. O reconhecimento do estresse ocupacional pelos gestores das instituições de saúde é necessário para que possam promover a qualidade de vida no trabalho e para que realizem ações de forma humanizada com o trabalhador, proporcionando um melhor ambiente de trabalho, minimizando o adoecimento e, consequentemente, proporcionando melhor produtividade e mais segurança ao paciente, além de respostas positivas à organização. Com esse estudo, entende-se ser necessária a realização de pesquisas que abordem as características dos trabalhadores da enfermagem e que colaborem com evidências científicas que abordem alternativas para amenizar e/ou solucionar os sinais do esgotamento profissional.

\section{Referências}

1. Lins RN, Collet N, Vaz EM, Reichert AP. Percepção da equipe de Enfermagem acerca da humanização do cuidado na UTI Neonatal. Rev Bras Ciênc Saúde. 2013;17(3):225-32.

2. Santini AM, Costenaro RG, Medeiros HM, Zaberlan C. Estresse: vivência profissional de enfermeiros que atuam em UTI neonatal. Cogitare Enferm. 2005;10(3):14-22.

3. Alves ME. Síndrome de Burnout. Psychiatry On-Line Brasil. 2017;22(9).

4. Sousa MV. Síndrome de Burnout em profissionais da saúde: estudo bibliográfico. Picos. Monografia [Trabalho de conclusão de curso] - Universidade Federal do Piauí; 2012.

5. Maslach C, Leiter MP. New insights into burnout and health care: Strategies for improving civility and alleviating burnout. Med Teach. 2017;39(2):160-3.

6. Cimiotti JP,Aiken LH, Sloane DM, Wu ES. Nurse staffing, burnout, and health care-associated infection. Am J Infect Control 2012;40(6):486-90.

7. Halbesleben JR, Wakefield BJ, Wakefield DS, Cooper LB. Nurse burnout and patient safety outcomes: nurse safety perception versus reporting behavior. West J Nurs Res. 2008;30(5):560-77.

8. Fernandes LS, Nitsche MJ, Godoy I. Associação entre síndrome de burnout, uso prejudicial de álcool e tabagismo na enfermagem nas UTIs de um hospital universitário. Ciênc Saúde Coletiva. 2018;23(1):203-14.

9. Gomes TR, Santos AF. A relação mãe-bebê prematuro na UTI neonatal: Um olhar Winnicottiano. REAS. 2020;12(2):e2422.

10. Paula CC, Padoin SM, Galvão CM. Revisão integrativa como ferramenta para tomada de decisão na prática de saúde. In: Lacerda MR, Costenaro RG (Org.). Metodologias da pesquisa para a enfermagem e saúde: da teoria à prática. Porto Alegre: Moriá; 2016. p. 51-76.
11. Souza MT, Silva MD, Carvalho R. Revisão integrativa: 0 que é e como fazer. Einstein (São Paulo). 2010;8(1):102-6.

12. Sexton JB, Sharek PJ, Thomas EJ, Gould JB, Nisbet CC, Amspoker AB, et al. Exposure to leadership walkrounds in neonatal intensive care units is associated with a better patient safety culture and less caregiver burnout. BMJ Qual Saf. 2014;23(10):814-22.

13. Profit J, Sharek PJ, Amspoker AB, Kowalkowski MA, Nisbet CC, Thomas EJ, et al. Burnout in the NICU setting and its relation to safety culture. BMJ Qual Saf. 2014;23(10):80613.

14. Soroush F, Zargham-Boroujeni A, Namnabati M. The relationship between nurses' clinical competence and burnout in neonatal intensive care units. Iran J Nurs Midwifery Rev. 2016;21(4):424-29.

15. Tawfik DS, Sexton JB, Kan P, Sharek PJ, Nisbet CC, Rigdon J, et al. Burnout in the neonatal intensive care unit and its relation to healthcare-associated infections. J Perinatol. 2017;37(3):315-20.

16. Melo LP, Carlotto MS. Programa de prevenção para manejo de estresse e síndrome de burnout para bombeiros: relato de experiência de uma intervenção. Estud Psicol. 2017;22(1):99-108.

17. Barros MM, Almeida SP, Barreto AL, Faro SR, Araújo MR, Faro A. Síndrome de burnout em médicos intensivistas: estudo em UTIs de Sergipe. Temas Psicol. 2016;24(1):377-89.

18. Boa Morte SV, Deps VL. Prevenção e tratamento do estresse e da síndrome de burnout em professores da rede pública de ensino. Interdiscip Scient J. 2015;2(1):62-75.

19. Tucker AL, Singer SJ. Estudo randomizado sobre uma intervenção baseada no programa leadership walkrounds. AHRQ. 2012;12-113.

20. Gasparino RC, Guirardello EB. Ambiente da prática profissional e burnout em enfermeiros. Rev Rene. 2015;16(1):90-6.

21. Lima FF, Alencar NE, Fernandes MA, Moura EC, Silva-Júnior FJ. Planejamento estratégico situacional em uma instituição psiquiátrica: contribuições e desafios. SMAD, Rev Eletrônica Saúde Mental Álcool Drog. 2019;15(2):20-8.

22. Barboza MC, Braga LL, Perleberg LT, Bernardes LS, Rocha IC. Estresse ocupacional em enfermeiros atuantes em setores fechados de um hospital de Pelotas/RS. Rev Enferm UFSM. 2013;3(3):374-82.

23. Carvalho CG, Magalhães SR. Síndrome de burnout e suas consequências nos profissionais de enfermagem. Revista da Universidade Vale do Rio Verde. 2011;9(1):200-10.

24. Dóro MP, Zampiron K, Okumura I, Andrzejevski VMS, Lolatto GA, Antonechen AC, et al. Interrelação entre Qualidade de Vida, Resiliência e Síndrome de Burnout: estudo Iongitudinal com residentes multiprofissionais. Revista Tempus Actas de Saúde Coletiva. 2018;12(1):83-100.

25. Reis LS, Silva EF, Waterkemper R, Lorenzini E, Cecchetto FH. Percepção da equipe de enfermagem sobre humanização em unidade de tratamento intensivo neonatal e pediátrica. Rev Gaúcha Enferm. 2013;34(2):118-24.

26. Padilha KG, Barbosa RL, Andolhe R, Oliveira EM, Ducci AJ, Bregalda RS, et al. Nursing workload, stress/burnout, satisfaction and incidents in a trauma intensive care units. Texto Contexto Enferm. 2017;26(3):e1720016.

27. Van Bogaert P,Timmermans O, Weeks SM, Van Heusden D, Wouters K, Franck E. Nursing unit teams matter: impact of unit-level nurse practice environment, nurse work characteristics, and burnout on nurse reported job outcomes, and quality of care, and patient adverse events - a cross-sectional survey. Int J Nurs Stud. 2014;51(8):1123-34.

28. Alves DF, Guirardello EB. Safety climate, emotional exhaustion and job satisfaction among Brazilian paediatric professional nurses. Int Nurs Rev. 2016;63(3):328-35.

29. França SP, De Martino MM, Aniceto EV, Silva LL. Preditores da síndrome de burnout em enfermeiros de serviços de urgência pré-hospitalar. Acta Paul Enferm. 2012;25(1):6873.

30. Andrade HS, Gomes HS, Mesquita GV, Ribeiro JL, Chagas FC. Prevalência de síndrome de burnout em pediatras intensivistas. R Interd. 2017;10(3):39-47. 